EPJ Web of Conferences 81, 06002 (2014)

DOI: 10.1051/epjconf/20148106002

(C) Owned by the authors, published by EDP Sciences, 2014

\title{
First results from the commissioning of the BGO-OD experiment at ELSA
}

\author{
Andreas Bella ${ }^{1, a}$ \\ for the BGO-OD Collaboration \\ ${ }^{1}$ Physikalisches Insitut, University of Bonn, Bonn, Germany
}

\begin{abstract}
The BGO-OD experiment at the ELSA accelerator facility in Bonn combines the highly segmented BGO calorimeter with a particle tracking magnetic spectrometer at forward angles. An extensive physics program using an energy tagged Bremsstrahlung photon beam is planned. The commissioning phase of the experiment is recently complete, enhancements for the BGO-OD experiment are nevertheless in development. Recent results from the analysis of the commissioning data, which includes particle track reconstruction in the forward spectrometer and momentum reconstruction with the BGO calorimeter are presented.
\end{abstract}

\section{Introduction}

The BGO-OD experiment is a fixed target experiment located at the electron strecher facility ELSA [1] at the University of Bonn. Its main goal is the study of photoproduction reactions off the nucleon, investigating simultaneously the contributions of the excited baryon resonances ( $s$-channel) and the ones related to intermediate particle exchange ( $t$-channel). A real photon beam is produced via the Bremsstrahlung process by using the provided electron beam from ELSA. The BGO-OD experiment consists mainly of the BGO calorimeter [2-4] and a magnetic spectrometer at forward angles. In this configuration the BGO-OD experiment is very well suited for the investigation of processes at low momentum transfer $t$ and it is optimised for the detection of mixed charged final states. This is important in the investigation of strangeness and vector meson photoproduction which are both $t$-channel dominated. An extensive physics program is planned which consists, for example, in the investigation of the $t$-channel exchange mechanism in $K^{0}$ and $K^{+}$photoproduction, measurements of the cross section (up to $3 \mathrm{GeV}$ ) and beam asymmetry (up to $1.7 \mathrm{GeV}$ ) for $\omega$ and $\phi$ photoproduction and of the beam asymmetry (up to $1.7 \mathrm{GeV}$ ) for $\eta$ and $\eta^{\prime}$ photoproduction. In addition, due to the high resolution measurement of the proton momentum in the forward spectrometer, the investigation of $\eta^{\prime}$ mesic states could reveal information about meson-nucleous interaction.

\section{Experimental setup}

The ELSA electron beam of energy $E_{0}$ is extracted into the experimental area and impinges on an amorphous (crystalline) radiator where an unpolarised (linearly polarised) photon beam is produced

\footnotetext{
ae-mail: bella@physik.uni-bonn.de
} 

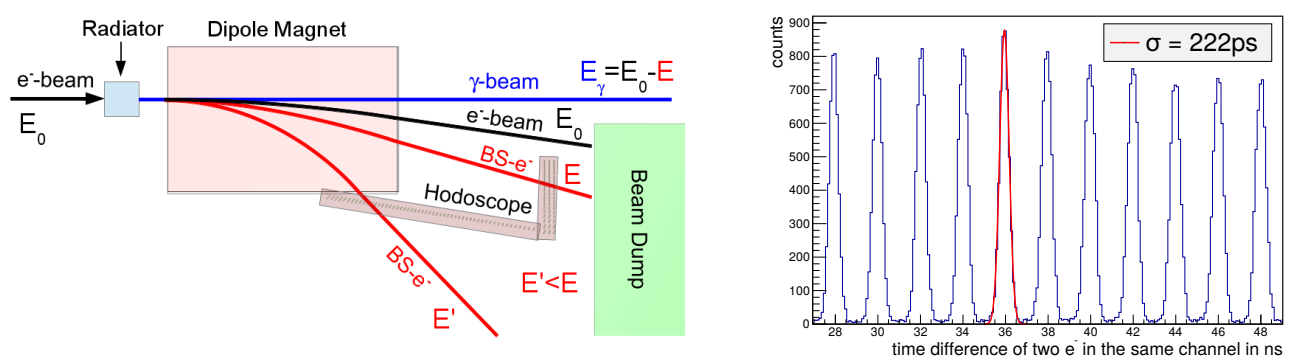

Figure 1. Left: Tagging principle. black: primary beam, blue: photon beam, red: Bremsstrahlung electrons. Right: Time difference of two electrons in the same tagger channel.

via Bremsstrahlung. The photon tagger [5,6] momentum analyses the Bremsstrahlung electrons by deflecting them in a dipole magnet before detecting them in a hodoscope (figure 1, left panel). The energy of the photon is then deduced from energy conservation, $E_{\gamma}=E_{0}-E$. The photon tagger detects Bremsstrahlung electrons whose energy ranges between $10 \%$ and $90 \%$ of $\mathrm{E}_{0}$ with a resolution from $0.16 \%$ to $0.62 \%$ of $E_{0}$. Figure 1 (right panel) shows the time difference between two electrons detected in the same tagger channel. It can be seen that the time resolution of the tagger is about 222 ps which allows to resolve the $2 \mathrm{~ns}$ bunch structure of ELSA. As a consequence, each electron can be assigned to the corresponding bunch. Since the bunch length is known to be equal to $80 \mathrm{ps}$, the event time is known with a precision of up to 80 ps.

The produced Bremsstrahlung photons impinge on a $\mathrm{LH}_{2}$ target surrounded by a central electromagnetic calorimeter. It consists of 480 Bismuth Germanate crystals covering an polar angular range from $25^{\circ}$ to $155^{\circ}$ and the full azimuthal angle, corresponding to a coverage of $0.9 \cdot 4 \pi$. A 32 strip scintillator barrel for charged particle identification and a MWPC for particle tracking are located inside the BGO calorimeter. The forward angles from $8^{\circ}$ to $25^{\circ}$ are covered by MRPC'S.

A forward spectrometer covers the polar angle up to $12^{\circ}$. It consists of two scintillating fibre detectors (MOMO and SciFi2 [7]) placed in front of an open dipole magnet and eight double layer driftchambers which are located behind the magnet. The dipole magnet is a MD type magnet on permanent loan basis from DESY. A magnetic field of $\mathrm{B}_{\max }=0.54 \mathrm{~T}$ is achieved with a maximum current of $\mathrm{I}_{\max }=1340 \mathrm{~A}$. Four walls with a size of $\sim 3 \mathrm{~m} \times 3 \mathrm{~m}$ of scintillating bars are installed behind the forward spectrometer for time of flight measurements and charged particle identification. The distance of the time of flight walls to the target amounts $\sim 5.5 \mathrm{~m}$. The experimental setup is completed with photon flux detectors.

\section{Preliminary results from the commissioning}

Data were taken in 2013 for the commissioning of the detector setup using both an unpolarised and a linearly polarised photon beam. This section presents an overview of these preliminary results.

Neutral meson reconstruction in the BGO ball: In figure 2, the invariant mass distribution of two photons in the BGO is shown. The two peaks correspond to the $\pi^{0}$ and the $\eta$ masses. Using a calibration based on the $1.27 \mathrm{MeV}$ photons from ${ }^{22} \mathrm{Na}$ sources, the fitted masses are in good agreement with the accepted values. Preliminary measurements of the absolute cross section of $\pi^{0}$ photoproduction off the proton has been performed and the results agree with the SAID PWA solution within the errors. 
MESON 2014 $-13^{\text {th }}$ International Workshop on Production, Properties and Interaction of Mesons

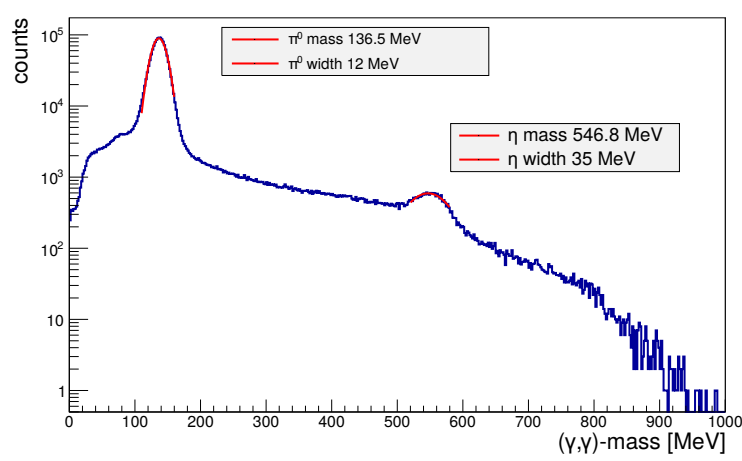

Figure 2. $(\gamma, \gamma)$ invariant mass spectrum. Peaks of $\pi^{0}$ and $\eta$ are visible.

First measurements with a linearly polarised photon beam: The use of a diamond radiator produces coherent Bremsstrahlung, resulting in a linearly polarised photon beam if the diamond radiator is properly aligned with respect to the primary electron beam. The alignment is performed through the stonehenge technique [9]. Figure 3 (left panel) shows a stonehenge plot of an aligned diamond radiator.
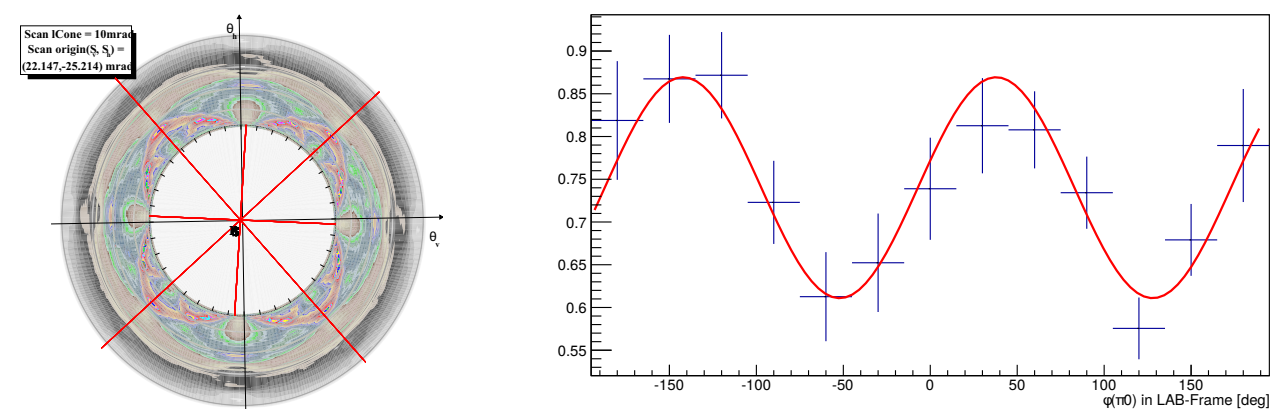

Figure 3. Left: Stonhenge plot of an already aligned diamond radiator. Fourfold symmetry and coherent Bremsstrahlung contribution of the (022) lattice vector are visible. Right: Azimuthal distribution of the ratio $\mathrm{N}_{\text {pol }} / \mathrm{N}_{\text {unpol }}$ in the incoming photon energy range $250 \mathrm{MeV}-400 \mathrm{MeV}$ in the reaction $\gamma \mathrm{p} \rightarrow \pi^{0} \mathrm{p}$ (blue - data points, red $-\cos (2 \phi)$ fit).

The fourfold symmetry as well as the dominant contribution to the coherent Bremsstrahlung of the (022) lattice vector is visible. $\pi^{0}$ photoproduction events have been identified, both in the case of a linearly polarised photon beam and for the unpolarised one. The ratio of the number of reconstructed events $\mathrm{N}_{\text {pol }} / \mathrm{N}_{\text {unpol }}$ is shown in figure 3 (right panel) for the incoming photon energies $\mathrm{E}_{\gamma}=250 \mathrm{MeV}$ $400 \mathrm{MeV}$ as a function of the azimuthal angle $\phi$ of the reconstructed $\pi^{0}$ in the laboratory frame. The expected $\cos 2 \phi$ distribution is observed.

Charged particle identification in the forward spectrometer: The magnetic spectrometer is used for charged particle identification for forward angles up to $\theta_{\text {hor }}=12^{\circ}$ and $\theta_{\text {vert }}=8^{\circ}$. For the momentum reconstruction a program called GENFIT [10] developed within the PANDA collaboration is used. Input parameters required by GENFIT are the reconstructed particle tracks, the geometry of the forward spectrometer and the magnetic field of the open dipole magnet. GENFIT considers multiple scattering and performs automatic energy loss corrections. Presently, a momentum resolution of about $2 \%$ is achieved. This will be improved in the near future using the drift time information 
of the drift chambers instead of the hit drift cell only. Combining the reconstructed momentum of the detected particles with the time information of the time of flight walls allows charged particle identification by determining the particle's velocity. Figure 4 (left panel) shows the speed of the charged particles as a function of their momentum. The distributions for $\pi^{+}$and $\mathrm{p}$ are clearly visible.

$K^{+}$identification in the BGO: The $K^{+}$identification in the BGO is performed through the reconstruction of its weak decay $K^{+} \rightarrow \mu^{+} v$. This technique has been already proven and exploited by the A2-collaboration [8] at MAMI. The missing mass calculated from the $K^{+}$detected in the BGO is shown in figure 4 (right panel) and both the $\Lambda$ and the $\Sigma^{0}$ peaks are visible.
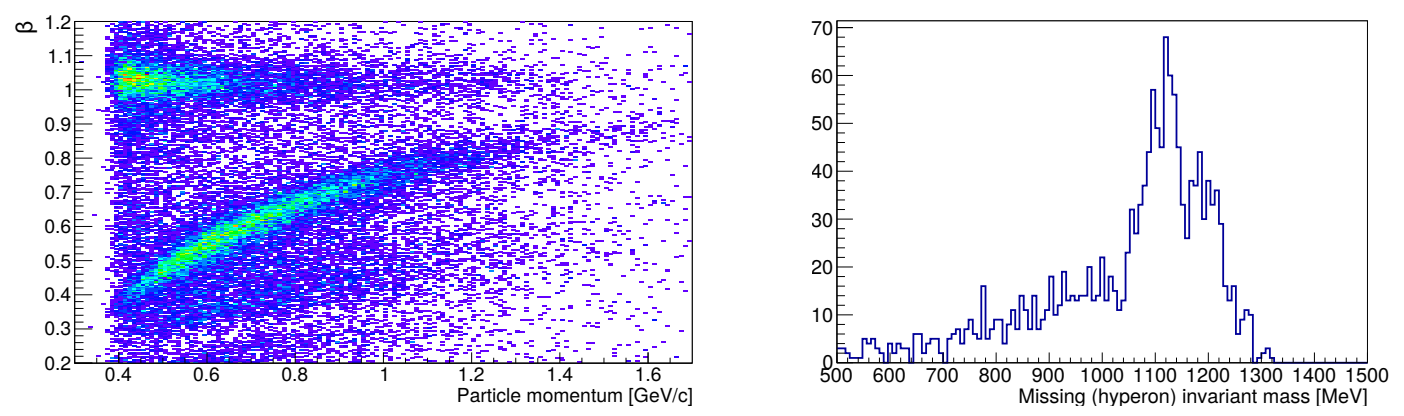

Figure 4. Left: Speed of charged particles as a function of their momentum. Data acquired for $\mathrm{C}$ target. Right: Missing mass from $\mathrm{K}^{+}$detection in the BGO ball.

\section{Conclusions}

The BGO-OD experiment is a unique experiment optimised for the detection of mixed charged final states. Due to the forward spectrometer it is well suited for the investigation of processes at low momentum transfer. The commissioning is already in an advanced stage but still ongoing. It shows already positive results in neutral and charged meson reconstruction in the BGO ball and the forward spectrometer using an unpolarised and polarised photon beam. The data taking will start soon after the commissioning of the newly installed MWPC.

\section{References}

[1] W. Hillert, Eur. Phys. J. A 28, 139 (2006)

[2] P. Levi Sandri et al., Nucl. Instr. Meth. Phys. Res. A 370, 396-402 (1996)

[3] F.Ghio et al., Nucl. Instr. Meth. Phys. Res. A 404, 71-86 (1996)

[4] M. Castoldi et al., Nucl. Instr. Meth. Phys. Res. A 403, 22-30 (1996)

[5] A. Bella, PhD Thesis in preperation, Rheinische Friedrich-Wilhelms-Universität Bonn

[6] F. Messi, PhD Thesis in preperation, Rheinische Friedrich-Wilhelms-Universität Bonn

[7] S. Boese, PhD Thesis in preperation, Rheinische Friedrich-Wilhelms- Universität Bonn

[8] T.C. Jude, D.I. Glazier, D.P. Watts et al., Phys. Lett. B 735, 112-118 (2014)

[9] K. Livingston, Nucl. Instr. Meth. Phys. Res. A 603, 205-213 (2009)

[10] C. Hoppner, S. Neubert, B. Ketzer, S. Paul, Nucl. Instr. Meth. Phys. Res. A 620, 518-525 (2010) 